\title{
ON THE CHOICE OF METHOD TO CANCEL 60 HZ DISTURBANCES IN BEAM POSITION AND ENERGY*
}

\author{
R. A. Akogyeram ${ }^{\dagger}$, R. W. Longman, Columbia University, New York, NY 10027 \\ A. Hutton, Jefferson Lab, Newport News, VA 23606 \\ J.-N. Juang, NASA Langley Research Center, Hampton, VA 23681
}

\begin{abstract}
Because the voltage applied to magnets in accelerators is likely to be rectified, there can be $60 \mathrm{~Hz}$ related fluctuations in beam position and energy. Correcting such errors as well as other less repeatable errors can be done with combinations of feedback, feedforward, real time repetitive control, and batch update repetitive control. This paper studies how to mix these approaches for optimized performance. It is shown that use of feedback control can be counterproductive because of the waterbed effect operating on errors such as BPM noise. Also, it is seen that iterative repetitive control updates can produce significantly better error levels than pure feedforward control. Making corrections of errors for harmonics of 60 $\mathrm{Hz}$ that are above the Nyquist frequency can be accomplished, and this can save the expense and integration effort to produce fast beam sampling.
\end{abstract}

\section{INTRODUCTION}

The DC current needed to operate electromagnets in accelerators may come from rectified AC. This process is imperfect and there is a small amount of residual fluctuation in the resulting voltage, containing $60 \mathrm{~Hz}$ and possibly many harmonics. These fluctuations translate into periodic errors in beam horizontal and vertical positioning as well as fluctuations in beam energy. Figure 1 gives an example time history of the horizontal beam position error, and Fig. 2 gives the corresponding frequency spectrum of such position errors in the $4.0 \mathrm{GeV}$ continuous electron beam accelerator at the Thomas Jefferson National Accelerator Facility. The 60, 120, and $180 \mathrm{~Hz}$ peaks not shown on the plot are at 315,16 , and 83 units on the DFT (corresponding to $0.0771,0.0039$, and $0.0203 \mathrm{~mm}$, respectively). This paper examines the possible ways to attenuate these errors together with any other non-periodic errors, and makes recommendations on the preferred approach. One approach is represented by the combined feedback and feedforward system operating at $2.4 \mathrm{kHz}$ in two of the three experimental halls of the Jefferson $4.0 \mathrm{GeV}$ accelerator [1]. The system aims to eliminate general errors in the range from 0 to $80 \mathrm{~Hz}$ as well as 60,120 , and $180 \mathrm{~Hz}$ components using the feedback system. The feedforward system aims to eliminate harmonics from 240 to $720 \mathrm{~Hz}$. This paper

\footnotetext{
* Work was supported by the U.S. D.O.E. under contract \#DE-AC0584-ER40150.

† Email: akogyera@jlab.org
}

summarizes the work reported in [2-4] that examines the range of possible approaches to see which should give optimized performance.

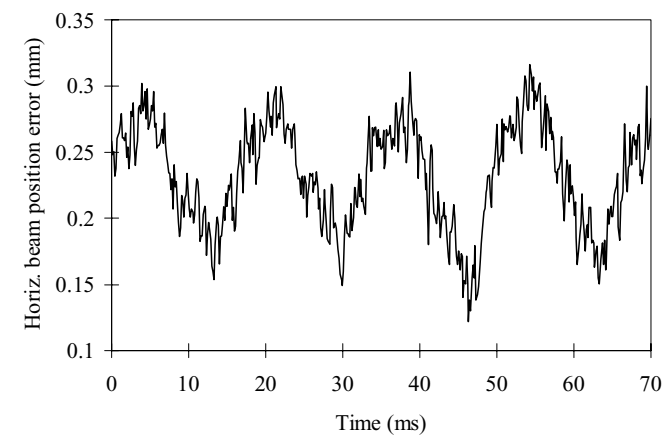

Figure 1: A periodic output error due to $60 \mathrm{~Hz}$ disturbance from the Jefferson Lab's accelerator.

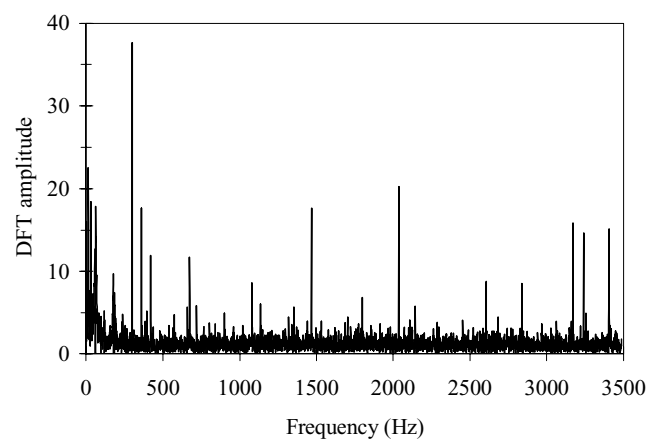

Figure 2: Frequency content of the horizontal beam position error with 60,120 , and $180 \mathrm{~Hz}$ peaks deleted.

\section{THE RANGE OF APPROACHES}

The range of possible approaches to address beam disturbances in position and energy include: 1. Typical feedback control. 2. Specialized feedback control that targets certain frequencies. 3. Real time repetitive control. 4. Pure feedforward control. 5. Batch update repetitive control. 6. Mixed feedback and feedforward. 7. Mixed feedback and batch repetitive control.

\section{WATERBED EFFECT}

Consider a standard unity feedback control system with a command going into a summing junction that takes the command and subtracts the feedback measurement to determine the error. The error goes into the control law that determines the corrective action, which is then 
applied to the system. The measured response is fed back to the summing junction closing the loop. In such a control system, the transfer function from command to resulting error, from a process disturbance modeled as an additive disturbance on the system output before feedback to resulting error, and from an additive measurement disturbance to resulting error are all the same (to within a sign). A basic result for the behavior of this transfer function is the Bode integral theorem [5], which says that if the error is attenuated by the feedback control action in one frequency range, the error must be amplified in some other frequency range. The waterbed effect applies to all of the approaches described above, except for pure feedforward and batch update repetitive control when harmonic decomposition of the error signal is applied carefully. Feedforward and batch repetitive control can only target the repeatable harmonic errors. Feedback control can target both non-repeatable disturbances as well as harmonic errors. However, handling the harmonic errors in feedback makes the correction subject to the waterbed effect, and hence must be paid for by amplification of errors in some other frequency range. Hence, an optimizes split in the control approach uses feedback for only the non repeatable errors, and all repeating harmonic disturbances should be addressed by either feedforward or batch update repetitive control.

\section{FEEDBACK CONTROL CAN BE COUNTERPRODUCTIVE}

A testbed was developed at Jefferson National Accelerator Facility to test possible beam control approaches [1] as shown in Fig. 3.

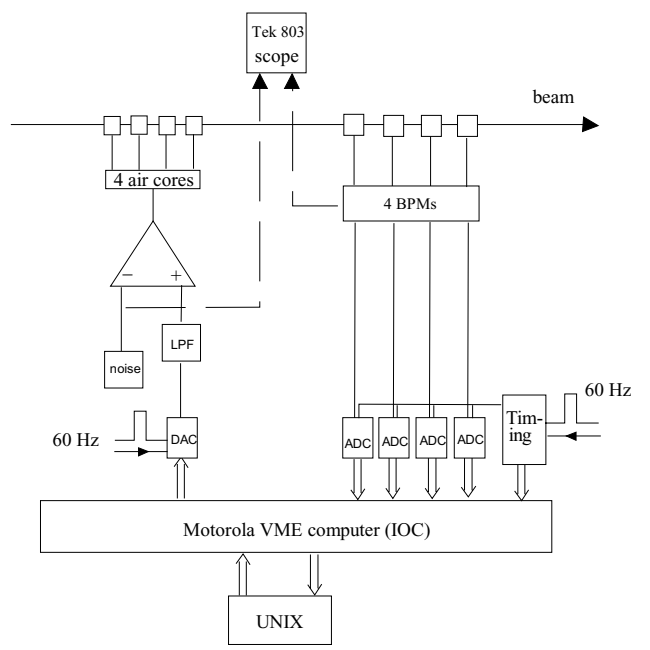

Figure 3: Hardware layout of the testbed.

It includes four air-core magnetic correctors, four BPMs, an operational amplifier with two input ports and one output port, a beam produced by electronics, and an arbitrary function generator connected to the BPMs. One of the two input ports is connected to a digital-analog converter (DAC) with an analog low pass filter (LPF) to smooth the DAC voltage synchronized to the power supply. The testbed was used without any feedback control, and then the Jeffereson feedback control system
[1] was turned on. After removing the $60 \mathrm{~Hz}$ and harmonics peaks from each, the error spectrum before and after are shown in Figs. 4 and 5. The RMS error is computed in each case, and it is seen that turning on the feedback control has increased the RMS error by $50 \%$ (rather than decreased it). This can be a result of the waterbed effect operating on BPM noise or on plant noise. Analogous results on the accelerator have not been generated.

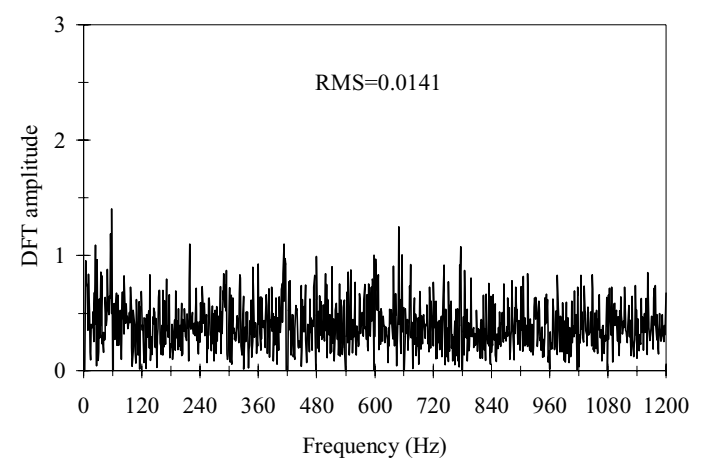

Figure 4: The frequency spectrum of the position error with the $60 \mathrm{~Hz}$ harmonics removed and without control.

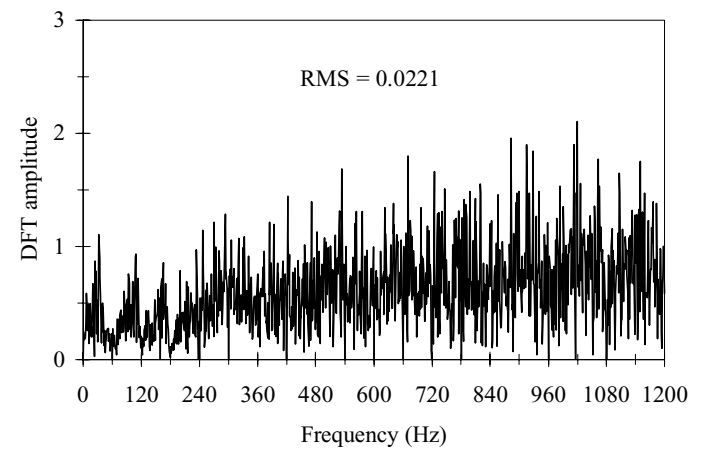

Figure 5: The frequency spectrum of the position error with the $60 \mathrm{~Hz}$ harmonics removed and with the notching feedback control running.

\section{FEEDFORWARD VS. BATCH REPETITIVE}

In a pure feedforward correction, the amplitude and phase of the $60 \mathrm{~Hz}$ and harmonics components of the error is found. Then based on knowledge of the amplitude and phase change from command to response for each frequency a corrective signal is applied. No additional correction is made to address any remaining error at these frequencies. A batch update repetitive control will repeatedly examine the error that remains, and try to correct it in the same manner. In the first case the final error level is determined by the accuracy in determining the harmonic components as well as the error in the input output model at the various frequencies. In the later case, provided the repeated corrections converge, the latter error source is eliminated, and the periodic updates allow tracking of drifts in the signal. This can be important. In 
either case, the accuracy of the corrections is limited by the accuracy in finding the harmonic components, and care should be taken to get these components as accurately as possible, for example by eliminating leakage effects [2]. Figure 6 shows the frequency contents of position error on the testbed when disturbances at 4 frequencies are injected. When a pure feedforward approach was used, based on a least squares decomposition of the error, the resulting position error is shown in Fig. 7 giving incomplete cancellation. When a batch repetitive control was applied, incorporating an iterative correction of remaining errors, then the error spectrum in Fig. 8 was obtained after 8 iterations.

\section{CONTROLLING ABOVE NYQUIST}

The $60 \mathrm{~Hz}$ related disturbances contain significant harmonic contents at high frequency as seen in Fig. 1. Upgrading was required at Jefferson in order to reach a $1200 \mathrm{~Hz}$ Nyquist frequency. Even at this $2400 \mathrm{~Hz}$ sample rate one must examine to be sure that no substantial aliasing of harmonics above Nyquist will occur. Using a sample rate that creates an integer number of sample times in the period of $60 \mathrm{~Hz}$ is important to eliminate leakage or nonorthogonality in determining the harmonic components of the error. But by deliberately using a sample rate that does not have this property, one can use a slow sample rate, and still control errors above Nyquist, by ensuring folded frequencies of interest are not folded onto another frequency of interest. An arbitrary function generator operating at a fast rate is required. This option is discussed in some detail in [3].

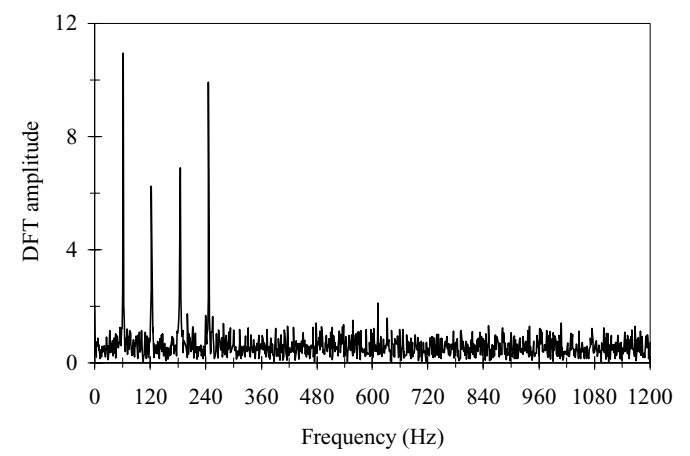

Figure 6: Frequency content of position error without correction.

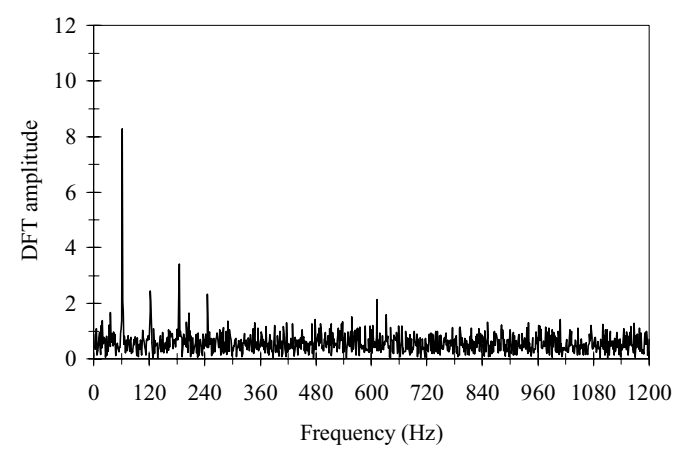

Figure 7: Frequency content for pure feedforward update.

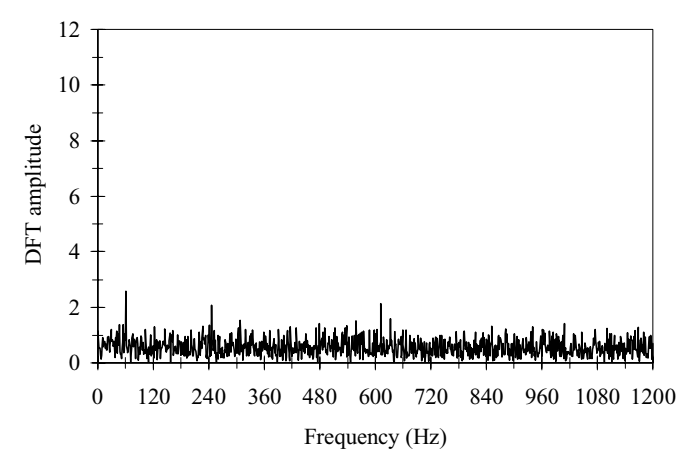

Figure 8: Frequency contents after 8 repetitions of batch repetitive control.

The authors are grateful to $R$. Dickson for setting up the experimental fixture.

\section{CONCLUSIONS}

Feedback control can be counterproductive because of the waterbed effects. An optimum cancellation can be obtained when feedback suppresses only non-periodic errors and feedforward or batch repetitive update cancels repeating errors. It is possible to correct harmonic frequencies above Nyquist by using an arbitrary function generator at a fast rate. Batch update repetitive control is not subject to waterbed effects, and cancellation does not require good estimates of the plant model. Pure feedforward control is also not subject to waterbed effect, but cancellation requires good estimates of the plant model and harmonics must remain unchanged with time.

\section{REFERENCES}

[1] R. Dickson, and V. A. Lebedev, "Fast Digital Feedback System for Energy and Beam Position Stabilization," Proc. of the 1999 Particle Accelerator Conference, New York City, NY, USA, March 29-April 2, 1999, Vol. 1, pp. 646-648.

[2] R. W. Longman, R. A. Akogyeram, A. Hutton, and J.N. Juang, "Stability of Matched Basis Function Repetitive Control," Advances in the Astronautical Science Vol. 105, 2001, pp.33-52.

[3] R. W. Longman, R. A. Akogyeram, A. Hutton, and J.N. Juang, "Control Law Design for Eliminating Periodic Disturbances above Nyquist Frequency," Proc. of the AIAA/AAS Astrodynamics Conference, Denver, CO, August 2000, pp. 418-432.

[4] R. W. Longman, R. A. Akogyeram, A. Hutton, and J.N. Juang, "Trade-offs between feedback, feedforward, and repetitive control for systems subject to periodic disturbances," Proc. of the AIAA/AAS Astrodynamics Conference, Santa Barbara, CA, February 2001, AAS 01194, to appear.

[5] T. Songchon and R. W. Longman, "On the Waterbed Effect and Repetitive Control using Zero-Phase Filtering," Proc. of the AIAA/AAS Astrodynamics Conference, Santa Barbara, CA, February 2001, AAS 01-196, to appear. 